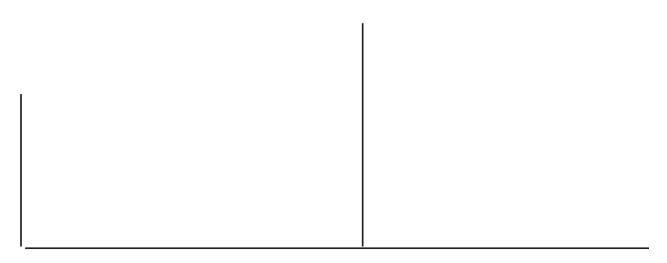

Rev. Latinoam. Psicopat. Fund., X, 3, 454-466

\title{
Fragments, constructions et destins de la honte dans la mélancolie
}

\author{
Delphine Scotto di Vettimo \\ Jean-Michel Vives
}

L'évocation clinique d'un jeune homme hospitalisé pour décompensation mélancolique montre l'importance de la prise en compte du vécu de honte qui lui est contingente. Cet affect de honte va lui permettre une réappropriation subjective de son expérience mélancolique et en accompagner la perlaboration. L'hypothèse ici envisagée postule que dans le travail clinique, l'expression de la honte comme mode d'appel au regard de l'Autre constitue une tentative du sujet de s'éprouver comme tel. Les auteurs questionnent l'expérience de honte dans la mélancolie et plus généralement l'évolution des conceptions de cet affect et d'un certain courant de pensée dans la psychanalyse aujourd'hui.

Mots clés: Narcissisme, mélancolie, pulsion invocante, sentiment de honte 
[...] Le regard prélève son objet sur une étendue, quitte à y inscrire son "hors-monde", tandis que la voix s'élève d'emblée sur fond d'un hors-monde, en quelque sorte: c'est là la fonction du silence. Mais, précisément, $l$ a découpe de la voix sur le silence pourrait bien faire miroirlécho à la découpe du regard, entre visible et invisible. Sur cette ontologie vocale du silence, le psychotique fait autorité d'expérience.

(Paul-Laurent Assoun, 1995, p. 5)

\section{Préambule}

L'une des difficultés majeures à la compréhension de la honte est due à son statut particulier et controversée dans la psychanalyse. En tant qu'affect, la honte peut se redoubler en "honte de la honte", être masquée, déplacée, condensée, déniée au même titre que d'autres affects négatifs et ce dans des constructions psychiques diversifiées. Elle entretient des rapports contrastées avec les représentations que l'on pourrait appeler communément "scènes de honte" et qui mettent en cause les sentiments de fierté et de dignité de même que les représentations qui leur sont liées; ces dernières pouvant rester secrètes ou devenir publiques, liées à une humiliation impliquant le rejet, l'exclusion et la perte d'amour. De la même manière, la honte peut aussi qualifier une action jugée déshonorante, du fait même du regard intérieur que le sujet porte sur lui-même et qui peut ou non être projeté sur le regard de l'autre. Autrement dit, la problématique de la honte s'inscrit d'emblée dans son rapport à autrui. Ce postulat nous conduit à interroger ici l'organisation d'un tel processus dans le champ de la mélancolie, plus particulièrement chez de jeunes adultes hospitalisés pour décompensation mélancolique et qui expriment - au cours des entretiens - un vécu de honte, d'infériorité inhérents à "leur maladie mentale", à "leur handicap" et à ses radicales conséquences.

Dans cette perspective, et ce sera ici notre propos, il convient de questionner la honte comme forme d'expression narcissique: le sujet trouverait un accès à la subjectivation dans et par l'épreuve de honte. 


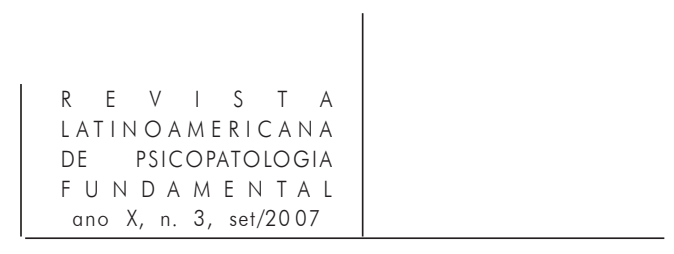

Cette assertion s'inscrit dans le prolongement d'une réflexion élaborée, d'une part à partir de la question du statut métapsychologique de la honte dans l'œuvre freudienne, de ses manifestations et de son traitement psychothérapique (Di Vettimo, 2001), d'autre part à partir de la question de l'appréhension de la honte dans la mélancolie. Nous proposons, à partir d'une situation clinique, de questionner l'épreuve de honte chez un sujet mélancolique et plus largement d'interroger la problématique de la honte, en regard du versant social où elle fait symptôme.

\section{Position du problème}

Le Dictionnaire de la langue française d'Émile Littré propose la définition suivante de la honte: "Déshonneur, honte, humiliation" et reprend aussi, en termes quasi similaires, la définition du Dictionnaire de l'Académie française qui s'énonce ainsi: "Confusion, trouble, sentiment pénible excité dans l'âme par l'idée de quelque déshonneur qu'on a reçu ou qu'on craint de recevoir, ou qu'on aurait seulement à ses propres yeux". Il convient de noter que la distinction entre le psychique et le social n'est pas clairement établie, distinction qui est capitale dans l'étude de la honte. L'usage de termes tels que "confusion", "sentiment" et "crainte" souligne le caractère psychique de la honte par rapport à son caractère social. En tant qu'affect, la honte est d'emblée sociale, étroitement liée au regard de l'autre, tout autant qu'expression de la manifestation d'une revendication singulière. Toute la question posée par la honte réfère bien à son caractère fondamentalement intersubjectif, à savoir qu'elle accompagne un échec devant témoin et conduit à vouloir le cacher.

La référence à la honte n'est pas rare dans l'œuvre de Freud et s'appréhende globalement comme indice d'un fonctionnement sur le mode narcissique. Dans $L^{\prime}$ 'interprétation des rêves $(1900)^{1}$ Freud souligne le caractère spéculaire de la honte, qui tient au regard: la honte se nourrit de l'incomplétude imaginaire qui constitue l'objet même de l'intérêt et de l'amour narcissique, en référence à une

1. Dans le texte de la Genèse, la naissance du sentiment de honte est contemporaine de la découverte de la nudité: Adam et Ève, après avoir commis le péché originel, se découvrirent nus... et honteux. La théorie psychanalytique freudienne fera du texte biblique le point de départ du sentiment de honte, comme celui de l'angoisse et de la culpabilité: “C'est pourquoi - note Freud dans L'interprétation des rêves (1900, p. 213) - dans le paradis les hommes sont nus et n'ont point de honte, jusqu'au moment où la honte et l'angoisse s'éveillent, où ils sont chassés et où commencent la vie sexuelle et la civilisation". 


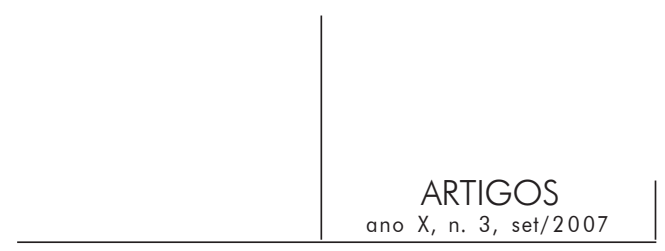

représentation de soi et de l'épreuve que représente le fait de se reconnaître imparfait, limité, manquant, c'est-à-dire soumis à l'épreuve de la castration. Audelà, dans l'œuvre de Lacan, la honte est considérée comme ontologique puisqu'elle est articulée à l'Homme. Cette hypothèse paraît s'accorder avec l'hypothèse phylogénétique freudienne sur la honte. À l'appui de ces considérations, l'émergence de honte ne porterait donc pas seulement sur le sentiment de valeur et d'estime de soi mais aussi et surtout sur le sentiment d'identité, dont la honte en révèlerait les fissures et les ruptures. C'est précisément dans cette occurrence qu'il s'agit de penser l'existence de honte chez le mélancolique. Cette conviction constitue une ligne d'écoute du discours de sujets confrontés à une expérience mélancolique et hospitalisés en psychiatrie. Quand une parole peut être énoncée à sa suite, on peut constater qu'elle se déploie dans le transfert pour dire une sorte d'indigence.

Parler de sa problématique mélancolique est alors avant tout se dire différent, décalé, dévalorisé et irrémédiablement abîmé. Par ailleurs, des expressions telles que étrangeté, errance, exclusion émaillent les entretiens avec de tels patients; autant d'expressions qui témoigneraient de ce recouvrement de la réalité par un réel qui submerge l'appareil psychique et ses constructions imaginaires et qui signent, à plus ou moins brève échéance, la décompensation mélancolique.

Cette involution inhérente à l'entrée dans la mélancolie perdrait le sens de ce qui, dans l'éprouvé de honte, fonctionnerait comme trace, soutenant ainsi l'essentiel de son enjeu subjectif, soit sa survenue comme sauvegarde narcissique.

Dans son ouvrage intitulé Le préjudice et l'idéal (1999) Assoun propose de considérer - “... des exilés au sens propre - délocalisation géographique et sociale - et des exilés au sens figuré - désubjectivation et malaise de l'intériorité” (p. 114). C'est précisément ce dernier argument qui nous intéressera ici au titre de ce qu'il révèle de l'expérience mélancolique comme épreuve de désubjectivation et d'exil intérieur. Comment un sujet peut-il être confronté, de l'intérieur, à ce sentiment d'être ailleurs, étranger, exilé? Une clinique de l'exil dans le champ de la mélancolie pourrait interroger cette épreuve d'étrangeté qui tient à une position subjective. Parler "d'exil intérieur" (ibid.) permettrait de désigner une sorte de métaphore, celle qui assigne le mélancolique à cette expérience - le plus souvent terrifiante - de dépossession de soi, d'exclusion et de désubjectivation.

Nous avancerons que cette articulation, qui trouve des fondements théoricocliniques, est renforcée par notre pratique de clinique analytique qui questionne régulièrement l'expression de sentiments de honte chez des sujets mélancoliques et qui surtout semblent envahir, invalider, l'ensemble de leur vie psychique, de leur expérience relationnelle et sociale. Non seulement la honte gagne, envahit la sphère subjective, mais la persistance devient répétition dans la mise en scène de récits de situations productrices de honte pour ces sujets. Pourquoi l'expérience 


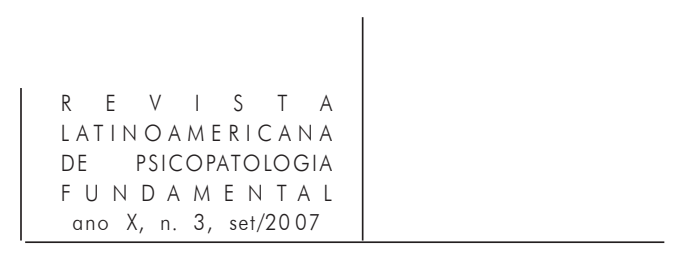

mélancolique produit-elle de la honte ? Sur cette question, notre hypothèse est que l'expression de la honte comme mode d'appel au regard de l'Autre constituerait une tentative du sujet de s'éprouver comme tel et de maintenir un lien social.

Nous proposons, dans cet article, de situer des développements et des connexions de cette prise de position à partir d'une situation clinique de rupture plus ou moins radicale: le discours psychopathologique d'un jeune homme qui traduit son entrée progressive dans un état mélancolique. Un extrait du travail thérapeutique engagé permettra d'une part de revenir sur notre hypothèse de travail et d'autre part de porter une attention particulière sur certaines modalités du processus mélancolique et de l'expérience de honte qui lui est contingente.

\section{Évocation clinique ${ }^{2}$}

\section{Données biographiques}

Christophe est un jeune homme de 18 ans, que j'ai suivi durant un an dans le cadre d'une psychothérapie analytique.

Il a été élevé par ses grand-parents paternels, qui ont servi de référents parentaux durant son enfance, à lui comme à son frère aîné. La mère a abandonné son mari et ses enfants alors que Christophe avait quelques mois. Le peu d'éléments historiques recueillis témoignent en faveur d'une "mère qui jetait son enfant sur la table à langer lorsqu'il fallait le changer", d'une "mère agressive et rejetante qui ne supportait pas son enfant".

Les troubles de Christophe sont apparus à l'adolescence, alors qu'il venait d'obtenir un C.A.P. de peintre en bâtiment, et se sont manifestés essentiellement sous forme de questionnements existentiels sur le vécu émotionnel, sur le sens de la vie etc. Le dossier de soins institutionnel mentionnait également une tendance à l'apragmatisme, des manifestations de perplexité par rapport à la réalité extérieure, sans que l'on puisse évoquer, à proprement parler, de vécu délirant.

Par contre, les troubles cognitifs, les troubles du cours de la pensée apparaissaient au premier plan d'une symptomatologie qui posait soit "la question d'une réaction dépressive massive ne permettant pas un fonctionnement intellectuel normal, soit d'une entrée dans une schizophrénie". Plusieurs hospitalisations se succéderont, en alternance entre l'hôpital psychiatrique et les cliniques psychiatriques privées.

2. La situation rapportée concerne l'un des auteurs de ce texte (Delphine Scotto Di Vettimo). Son élaboration après-coup a été faite par les deux signataires de l'article. 


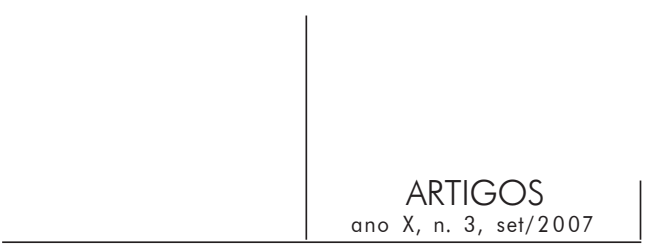

À l'occasion d'une nouvelle hospitalisation en raison d'un "état dépressif sévère avec manifestations de symptômes psychotiques", Christophe se donne un coup de couteau dans l'abdomen - alors que rien de notable ne prédisposait à anticiper un tel acte - et rationalise son geste en expliquant qu'il ne supporte plus les périodes d'amélioration de son état, qui sont toujours significatives pour lui de risques de rechute. De façon plus globale, il verbalise sa difficulté à pouvoir trouver un sens et un plaisir à la vie. À la suite de ce passage à l'acte auto-agressif, Christophe est hospitalisé d'urgence dans un service de soins intensifs. Fait important, c'est à cette occasion que sa mère, qu'il n'a plus vu depuis de nombreuses années, reprendra contact avec lui et viendra lui rendre visite.

Un mois plus tard, dès sa sortie du service de chirurgie digestive, Christophe est de nouveau hospitalisé dans le même service de psychiatrie. Quinze jours plus tard, toujours dans sa chambre, il commet un nouveau passage à l'acte suicidaire: cette fois-ci, il se tire une balle de 22 long rifle dans la poitrine. Il est de nouveau hospitalisé d'urgence dans le même service de chirurgie digestive. Le psychiatre qui lui rend visite note "une dimension mélancolique manifeste qui n'était pas apparue jusqu'à ce jour, caractérisée par des idées d'incurabilité, un désir de mort répété, une souffrance très vive". À la suite de ce deuxième passage à l'acte, sa mère se manifeste une nouvelle fois et lui rend visite; les rencontres entre la mère et le fils se sont avérées difficiles, Christophe disant ne pas pouvoir avoir de gestes de tendresse vis-à-vis de cette femme qui a été très rejetante dès les premiers mois de sa vie. Dans les semaines qui suivent, il se rétablit progressivement et ses jours ne sont plus en danger.

Six mois vont s'écouler, durant lesquels l'acuité de la symptomatologie dépressive semble s'amenuiser. L'hypothèse diagnostique médicale retenue est la suivante: "Épisode dépressif sévère contingent d'une entrée dans un état mélancolique". Après un an d'hospitalisation en psychiatrie et compte tenu de l'amélioration clinique du patient, l'Équipe de soins suggère la mise en place d'un projet de réinsertion sociale et professionnelle.

Le projet thérapeutique nouvellement proposé consiste en une prise en charge journalière dans un atelier thérapeutique et au maintien de l'hospitalisation de nuit dans le service.

\section{Récits cliniques}

Alors que la prise en charge à l'atelier thérapeutique débute à peine, Christophe sollicite des entretiens car il a le sentiment que sa vie entière est un échec généralisé et cette conviction le fait vivre dans la terreur d'exploser, dans 


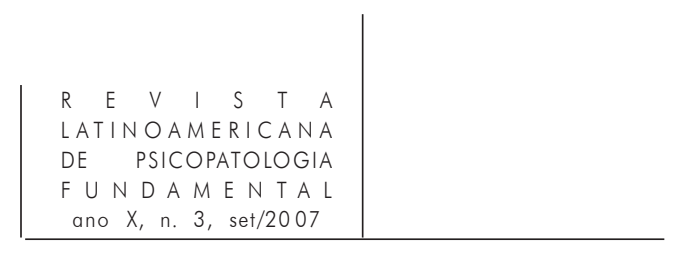

une douleur aiguë et permanente, avec le sentiment d'une menace constante sur son intégrité qu'il évoquera en ces termes lors du premier entretien: J'ai peur d'y laisser ma peau. D'emblée je le sens mal à l'aise: nous sommes assis en face à face et Christophe ne me regarde pas, son visage et ses yeux étant légèrement inclinés vers le bas et semblant regarder le parterre. Il dit, laconique: Médicalement, je suis soigné pour dépression. Mais mon problème majeur, c'est un problème de communication: j'ai du mal à m'exprimer. L'origine de ce problème, selon lui, est à relier sans conteste à la prise de toxiques, de "shit" en particulier, dont il a été pendant des années un fervent consommateur. Au cours de ce même entretien, il évoque des questionnements existentiels qui témoignent de sa tentative pour donner un sens à sa vie.

Enfin, mon attention s'éveille lorsqu'il dit, toujours les yeux baissés: Dans le silence, je ressens de la honte... je vais partir de n'importe quoi, Zorro, les Schtroumpfs, et développer. Ce qui compte, c'est d'éviter le silence pour éviter la honte. Le patient se plaint de lui-même, de ce qu'il est, de ce qu'il est devenu. Il reprend l'inventaire itératif et toujours péjoratif des éléments de son histoire, de ses attitudes, de ses défauts. Il aboutit au constat d'une sorte de malchance existentielle. Les faits rapportés s'inscrivent dans un climat de marasme, de désarroi et de malaise. Ils constituent autant d'éléments narratifs sur lesquels s'agrippe une angoisse sans nom: Je trouve cela inhumain répète-t-il. Il affirme ne plus parvenir à suivre une conversation normale et que plus que tout, c'est le silence qu'il redoute. Le constat de ces insuffisances débouche sur la conscience accrue et cruelle d'une défaillance irrémissible.

Dans les semaines qui suivent, il sera amené à développer le thème de souvenirs d'enfance et plus précisément à remémorer le douloureux sentiment d'avoir été - trop souvent - confronté à la honte et l'humiliation. Selon lui, vingt ans après, ce vécu infantile explique en grande partie sa tendance - dit-il - à avoir honte de tout. Enfin, il finira par associer directement la honte ressentie à sa maladie, qu'il évoquera de la manière suivante: La maladie joue un rôle très important dans l'humiliation et la honte que je ressens, car je me sens humilié et honteux dans le fait d'avoir des problèmes pour communiquer.

Quelques mois plus tard et alors que les entretiens se poursuivent à leur rythme habituel, Christophe abordera de manière plus précise les préoccupations touchant à l'image de soi et à sa fragilité narcissique: Je voudrais afficher une meilleure image de moi-même. Je me sens tellement ridicule. J'ai l'impression d'avoir le cerveau étroit. Je ne prends pas de plaisir dans la vie et je me sens aveugle. J'ai peur que les autres pensent de moi que je suis un handicapé mental. Il confiera que sa pensée interroge en permanence "l'avant" et "l'après" maladie mais surtout les effets irréversibles de perte, subjective et narcissique, que cette dernière a provoquée. Voici ce qu'il dit: Avec la maladie, j'ai conservé ma voix 


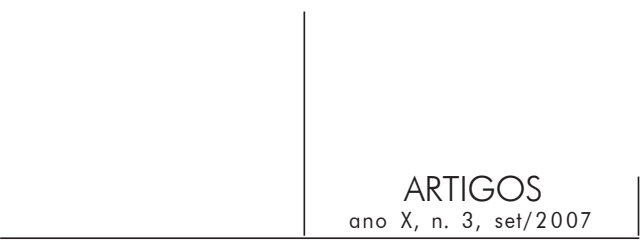

mais j'ai perdu mes capacités humoristiques et imaginaires. Cette dépression m'a tué... je ne vis plus comme les autres. Le désarroi psychique qu'entraîne cette prise de conscience d'une maladie irréversible, le dépouille ponctuellement de repères temporels et identitaires, où passé, présent et avenir se télescopent, où il ne sait alors plus qui il est. Et c'est à cette évocation qu'il réalise alors que c'est depuis cette dépression qu'il évite le silence pour éviter la honte: Depuis ma maladie, je parle beaucoup pour éviter qu'il y ait du vide ou du silence. Le silence, c'est une dévalorisation, c'est "la honte". Il faut que je maintienne un lien, un contact, je redoute la rupture du lien et l'abandon. Dans la rupture de lien, je suis dans la perte et le néant.

Cet extrait d'un travail clinique va nous permettre de revenir à présent sur l'hypothèse indiquée au début de cet article. À dessein, nous avons choisi d'interroger une des modalités du processus de honte qui mérite selon nous une attention particulière: la honte comme mode d'appel au regard de l'Autre.

\section{Du stade du miroir à l'Autre du regard}

Le stade du miroir marque une étape génétique et ontologique fondamentales, dans la mesure où va s'y constituer la première ébauche du moi. Face au miroir, l'enfant va anticiper, dans un leurre spéculaire et imaginaire, l'appréhension et la maîtrise de son unité corporelle. Son comportement face à son image dans le miroir se caractérise, nous dit Lacan (1945), par "L'assomption jubilatoire de son image spéculaire par l'être encore plongé dans l'impuissance motrice et la dépendance du nourrissage qu'est le petit homme à ce stade infans..." (p. 94). L'enfant perçoit dans l'image du semblable ou dans sa propre image spéculaire une forme (Gestalt) dans laquelle il va anticiper imaginairement l'appréhension et la maîtrise de son unité corporelle (Laplanche et Pontalis, 1967, p. 452). Cette unification va s'effectuer par l'identification de l'enfant à cette image: "Il y suffit de comprendre le stade du miroir comme une identification au sens plein que l'analyse donne à ce terme: à savoir la transformation produite chez le sujet, quand il assume une image" (Lacan, 1945, p. 94). C'est donc à partir de l'autre semblable et de sa reconnaissance que l'instance du moi s'ébauche et se constitue. Cette expérience narcissique fondamentale constitue la matrice symbolique de ce qui sera le moi. Elle est au fondement de l'expérience imaginaire du moi, constitué d'emblée comme "je-idéal" et "souche des identifications secondaires" (ibid.). Le début de cette structuration subjective caractérise le narcissisme primaire, c'està-dire l'investissement pulsionnel du sujet sur lui-même, sur cette image de lui à laquelle il s'identifie. En ce sens, la relation intersubjective, qui est marquée des 


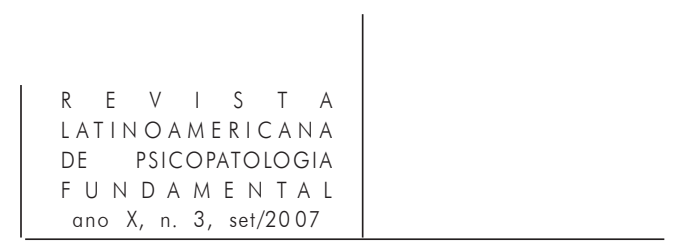

effets du stade du miroir, est d'abord une relation imaginaire et duelle, où le moi est constitué comme un autre et autrui comme alter ego. Narcissisme qui est la forme d'investissement inaugural pulsionnel indispensable à la vie, et en tant que tel, une donnée structurale du sujet.

Dans cette construction de l'image, nous faisons l'hypothèse que le patient a rencontré un regard vide, regard de l'Autre maternel dépouillé de toute résonance vitale et désirante. C'est-à-dire que l'Autre maternel, investi de la toutepuissance au temps de l'enfance, n'est pas venu le combler, lui, infans assujetti à son désir. En d'autres termes, il n'a pas rencontré d'intention signifiante dans le regard maternel qu'il n'a pas lu: le regard était vide car non articulé à une parole. Regard maternel qui, tourné vers sa propre béance, a exclu l'enfant de ce champ de reconnaissance. Ce qui amène "... à envisager le rapport du mélancolique à l'autre primordial comme marqué par une non-rencontre. L'autre a bien existé, mais lui-même préoccupé par autre Chose, semble avoir abandonné trop tôt l'enfant" (Vives, 2006, p. 312). Ainsi, de cette catastrophe première qui l'a précipité dans un désespoir sans cause, il n'y a pas de figuration possible car elle se situe en deçà du spéculaire et de la représentation. Nous allons voir, à l'appui de l'outillage conceptuel de Lambotte, que si le sujet est en faute, c'est de ne pas avoir été admis à adhérer à l'illusion identitaire dans laquelle se fondent les autres hommes, c'est-à-dire s'approprier son image et la prendre pour soi. Car il a été laissé pour compte de cette inscription première dans le désir maternel qui permet la rencontre du miroir. En ce sens, "le mélancolique serait moins privé d'une voix (comme peut se vivre le névrosé) ou envahi par la voix de l'Autre (comme cela peut être le cas pour le psychotique) que suspendu à un cri qui semble ne pas pouvoir se transformer en appel" (ibid., p. 313).

Dans ses remarquables études sur la mélancolie, Lambotte (1984) pose les principes métapsychologiques d'une nouvelle approche, qui allie - au modèle freudien de la "névrose narcissique" - des avancées possibles grâce à la notion de stade du miroir chez Lacan. Elle propose une ouverture psychogénétique de la maladie, qui s'originerait dans le défaut d'un regard maternel, absent ou insuffisamment désirant. Autrement dit, "tout se passe comme si une nonrencontre - ou pour le moins une rencontre problématique - entre la voix de l'infans et la parole de l'Autre, elle-même sous tendue par sa voix, rendait impossible la formulation d'une demande. Il s'agit moins ici d'une crainte de refus que de la certitude d'une fin de non recevoir frappant, par avance, toute demande de caducité" (Vives, 2006, p. 313). L'infans est traversé, sans être circonscrit, par ce regard littéralement appelé ailleurs, regard maternel impossible à capter, dont la terrifiante absence vient à s'illustrer toujours de manière traumatique dans les productions mélancoliques. J. Hassoun avait parfaitement repéré ce phénomène: "La mutité et l'aveuglement de l'Autre, son indifférence à l'adresse provoquent 


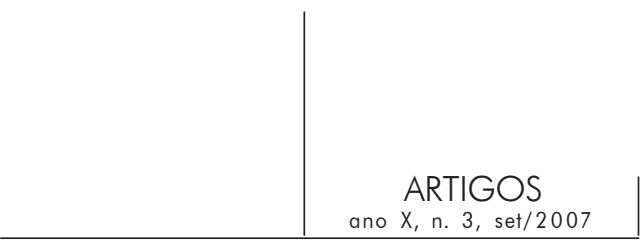

chez le sujet une sidération qui l'installe en deça du deuil. Nous pouvons dire des mélancoliques que quelque chose leur est arrivé, en ce sens que l'adresse de leur discours s'est perdue dans les limbes" (Hassoun, 1997, p. 58). Dès lors, c'est l'identification spéculaire de l'infans à son image corporelle qui va s'en trouver gravement compromise. Son reflet spéculaire ne sera plus pour lui qu'un cadre désespérément vide alors même qu'il reste rivé à un modèle maternel idéal, tout puissant et intangible.

Cette rencontre manquée du désir de l'Autre, et par là, de l'infini de ce qui aurait été l'objet de son désir, ne lui laisse en pâture que le rien. À la différence de la forclusion psychotique, la négation mélancolique trouve à s'exprimer dans le registre du langage et accompagne un comportement tout axé sur la mise à distance des investissements. La réalité n'est donc pas rejetée, au sens où le sujet n'en aurait jamais rien su, mais elle fait que le sujet s'identifie au "reste" de l'opération qui l'a constitué et se trouve marqué du signifiant "rien" qui caractérisera désormais son rapport à l'Autre. Le rien pour lequel il se prend, le rien du monde, le rien de l'Autre absent que le patient évoquait en ces termes: Dans la rupture de lien, je suis dans la perte et le néant. Ici, la négativité du discours mélancolique qui s'exprime dans le fait qu'il s'identifie au rien, qu'il se considère ruiné et dépossédé de tous ses biens, se trouve nécessairement dans un rapport symbolique avec un état nostalgique qu'il a suffisamment expérimenté pour ensuite rejeter ce qui pourrait n'en être qu'un pis-aller.

\section{Du silence vocal à la honte comme mode d'appel au regard de l'Autre}

Silence des soins maternels, silence vocal, silence dans la réclamation de soins et d'amour. Ainsi, le vide ressenti dans le silence ferait écho à ce même vide, antérieurement éprouvé, dans l'appel du petit enfant resté "lettre morte", sans destinataire maternel. Classiquement, "le cri de l'infans est entendu par la mère comme étant un appel où elle s'attache à lire une demande. C'est sa voix qui est interprétée comme signifiante. La voix est prise comme objet premier, comme objet perdu à partir du moment où la mère donne une signification à cette voix, la voix comme objet est perdu derrière ce qu'elle signifie pour l'Autre" (Vives, 2006, p. 311). À l'appui de ces considérations, nous formulons l'hypothèse que ce silence si redouté chez Christophe viendrait révéler la béance d'un espace non symbolisé, celui de son propre espace intérieur laissé "en friche" faute d'avoir rencontré chez l'Autre maternel de quoi désirer et être désiré. Pour le dire en d'autres termes, il s'agit de penser ici le silence comme accentuant l'objet voix. Entre le silence muet de l'impartageable et la nécessité tout aussi

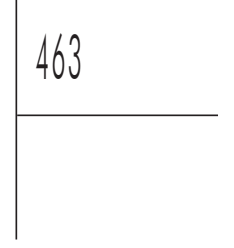




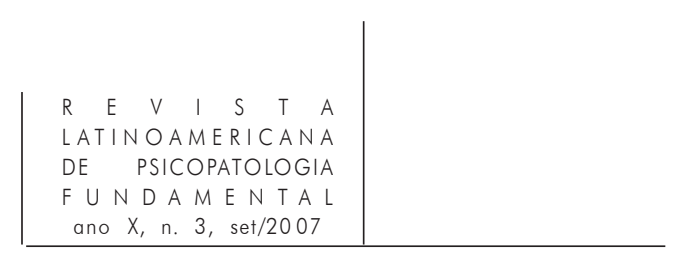

impétueuse de partager - Il faut que ça sorte avait-il dit un jour - se manifeste d'abord le silence comme révélant la béance d'un espace non symbolisé. Mais nous pouvons aller au-delà et postuler que si ce silence est aussi menaçant, c'est qu'il se confond, en son chaos imaginaire, avec le réel pur.

À dessein, la nécessité impérieuse de parler et le déchaînement vocal qui s'en suit - qu'il évoquait en ces termes - Je vais partir de n'importe quoi, Zorro, les Schtroumpfs, et développer viendrait pour parer à quelque chose qui est non symbolisable, ici le silence vocal maternel c'est-à-dire, en écho, l'absence réelle de l'Autre en son gouffre originaire. C'est en ce sens que le mélancolique tenterait de ne pas "se faire rien", c'est-à-dire perte et vide de la Chose. Alors que la dynamique invocante s'est trouvée invalidée au moment de la constitution même du sujet psychique, rendant problématique l'investissement de la réalité et l'articulation d'une demande.

En ce sens, pour le patient, donner de la voix deviendrait un impératif, sorte de matière sonore qui "troue" le silence comme ses propos en témoignent: "Ce qui compte, c'est d'éviter le silence pour éviter la honte". Assoun y fait très explicitement référence quand il écrit : “... la pulsion dite 'invocante' suppose de se faire voix pour quêter l'oreille de l'Autre" (1995, p. 85). Cette épreuve redoutée - de honte nous amène à déplier la scène originaire où l'objet s'est révélé irréparable. Abîmé par l'absence, le silence viendrait faire écho à l'impossibilité d'accéder jamais à l'objet et en accentuerait le drame. En ce sens, l'effroi du silence serait à entendre comme l'effroi du sans parole, qui vient faire vaciller sa propre image spéculaire, tout comme la parole, les mots la remettraient en place. En écho, l'invocation à la honte comme expérience intersubjective et comme mode d'appel au regard de l'Autre, permettrait de retrouver les voies d'un pensable, d'un figurable qui viennent en border le trou, tout en faisant lien commun avec un autre.

\section{Conclusion}

Notre propos a insisté, à travers l'évocation clinique d'un jeune homme hospitalisé pour décompensation mélancolique, sur cette co-occurrence entre honte, mélancolie et lien social. À dessein, nous avons choisi d'interroger ici la dialectique de la honte comme mode d'appel au regard de l'Autre. Au-delà, l'interprétation théorique et clinique proposée ici vise ici à postuler l'expression de honte comme le moyen pour être reconnu comme sujet et objet différencié, dans le sens où l'appropriation subjective de la honte marquerait et inaugurerait un seuil anthropologique et ontologique. 


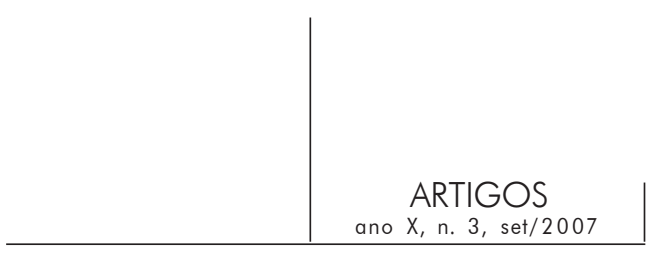

Références

Assoun, P.-L. Leçons psychanalytiques sur le regard et la voix. Tome 1: Fondements. Paris: Anthropos, 1995.

Leçons psychanalytiques sur le regard et la voix. Tome 2: Figures. Paris: Anthropos, 1995. 1999.

Le préjudice et l'idéal. Pour une clinique sociale du trauma. Paris: Anthropos,

Di Vettimo, D. Scotto (2001). Thèse pour le Doctorat en Sciences Humaines: Métapsychologie et clinique de la honte: son statut, ses manifestations, son traitement psychothérapique. Université de Nice Sophia-Antipolis.

Freud, S. (1900). L’interprétation des rêves. Paris: PUF, 1967.

Hassoun, J. La cruauté mélancolique. Paris: Flammarion, 1997.

LACAN, J. (1945). Le stade du miroir comme formateur de la fonction du Je. Écrits. Paris: Éditions du Seuil, 1966, p. 93-100.

Lambотte, M.-C. Esthétique de la mélancolie. Paris: Aubier, 1984.

Le discours mélancolique. Paris: Anthropos, 1993.

Laplanche, J. et Pontalis, J.-B. (1967). Vocabulaire de la psychanalyse. Paris: PUF, 1992.

Vives, J.-M. L'avocation mélancolique. Cliniques Méditerranéennes. Déclinaisons du monothéisme. Toulouse: Érès, n. 73, p. 303-17, 2006.

\section{Résumés}

O estudo clínico de um jovem homem hospitalizado por descompensação melancólica mostra a importância de levar em consideração a vivência da vergonha que lhe é contingente. Este afeto de vergonha vai permitir-lhe uma reapropriação subjetiva de sua experiência melancólica e acompanhar a sua perlaboração. A hipótese aqui encarada postula que no trabalho clínico, a expressão da vergonha como modo de atrair o olhar do Outro constitui uma tentativa do sujeito sentir-se como tal. Os autores questionam a experiência de vergonha na melancolia e, mais geralmente, a evolução das concepções destes afetos e de uma determinada corrente de pensamento na psicanálise hoje.

Palavras-chave: Narcisismo, melancolia, pulsão invocante, sentimento de vergonha 


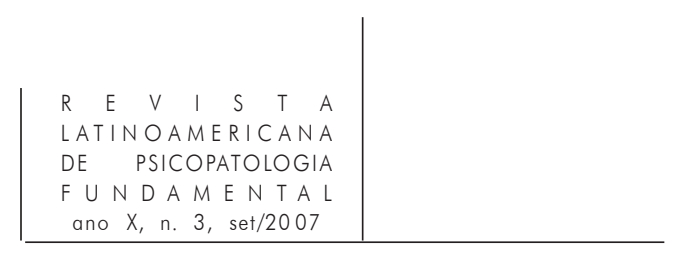

La evocación clínica de un joven hospitalizado por una descompensación melancólica muestra la importancia de llevar en cuenta la vivencia de vergüenza que le es contingente. Este afecto de vergüenza va permitirle un trabajo de reapropiación subjetiva de su experiencia melancólica y acompañar la reelaboración. La hipótesis aquí considerada postula que en el trabajo clínico, la expresión de la vergüenza como método de apelo al Otro constituye una tentativa del sujeto de probarse como tal. Los autores cuestionan la experiencia de vergüenza en la melancolía y más ampliamente en la evolución de las concepciones de ese afecto, así como una corriente de pensamiento en el psicoanálisis de hoy.

Palabras claves: Narcisismo, melancolía, pulsión de invocación, sentimiento de vergüenza

This clinical description of a young man hospitalized for a melancholic disturbance shows the importance of taking into account his contingent experience of shame. This affect of shame allows him to subjectively expropriate his experience of melancholia and accompanies his process of working it through. The assumption considered here postulates that in clinical work, the expression of shame as a way of calling to the Other constitutes an attempt for the question to be proven as such. The authors question the experience of shame in melancholia and, more generally, the evolution of the conceptions of affects and a certain current of thought in psychoanalysis today.

Key words: Narcissism, melancholia, invocating impulse, feelings of shame

Versão inicial recebida em abril de 2007

Versão revisada recebida em julho de 2007 